\title{
Multiple Shift Scheduling of Hierarchical Workforce with Multiple Work Centers
}

\author{
Salem M. AL-YAKOOB \\ Department of Mathematics\&Computer Science, College of Science, Kuwait University \\ P. O. Box: 5969 Safat 13060, State of Kuwait \\ e-mail:salem@al-yakoob.com \\ Hanif D. SHERALI* \\ Grado Department of Industrial\&Systems Engineering, Virginia Polytechnic Institute\& \\ State University, Blacksburg, VA 24061, USA \\ e-mail: hanifs@vt.edu
}

\begin{abstract}
This paper is concerned with an employee scheduling problem involving multiple shifts and work centers, where employees belong to a hierarchy of categories having downward substitutability. An employee at a higher category may perform the duties of an employee at a lower category, but not vice versa. However, a higher category employee receives a higher compensation than a lower category employee. For a given work center, the demand for each category during a given shift is fixed for the weekdays, and may differ from that on weekends. Two objectives need to be achieved: The first is to find a minimum-cost workforce mix of categories of employees that is needed to satisfy specified demand requirements, and the second is to assign the selected employees to shifts and work centers taking into consideration their preferences for shifts, work centers, and off-days. A mixed-integer programming model is initially developed for the problem, based on which a specialized scheduling heuristic is subsequently developed for the problem. Computational results reported reveal that the proposed heuristic determines solutions proven to lie within 92-99\% of optimality for a number of realistic test problems.
\end{abstract}

Key words: employee scheduling, manpower scheduling, mixed-integer programming,hierarchical workforce, scheduling algorithm.

\section{An Overview and Related Literature}

Hierarchical workforce scheduling problems arise in scheduling health-care personnel, job-shop employees, maintenance crews, and so on. In such problems, the employees are classified into different categories based on their educational background, training, and experience. An employee classified at a higher category can perform the duties of an employee classified at a lower category, but not vice versa. Consequently, an employee at a higher classified category usually receives a greater compensation than an employee at

\footnotetext{
* This work was supported by Kuwait University under Research Grant No. [SM06/02] and by the National Science Foundation under Research Grant No. [DMI-0094462]. Special thanks to Mrs. Fatima Al-awadi for her contributions to the computational implementation of the scheduling algorithm.
} 
a lower classified category. The demand requirements for employees of a given category are specified for weekdays and weekends, and the main objective is to meet these demand requirements using the most economical workforce mix of categories of employees. In this paper, we consider three shifts given as follows: 6:00 a.m. - 2:00 p.m., 2:00 p.m. 10:00 p.m., and 10:00 p.m. - 6:00 a.m. Required work features include: a) an employee may be assigned to at most one shift per day; b) each employee is entitled to two (not necessarily consecutive) off-days every week including a minimum specified number of off-days coinciding with weekends during the scheduling time-horizon if desired, and c) an employee can work for at most five consecutive days.

Due to the combinatorial nature of such problems, manual approaches to generate employee schedules are often too costly, inefficient, and time consuming. The utilization of formal modeling approaches to tackle such problems becomes imperative, especially, for large-scale hierarchical employee scheduling problems. Below, we discuss some literature related to the problem being considered in this paper.

One of the first formal approaches to this class of problems was proposed by Emmons and Burns (1991). Prior to this work, the emphasis of employee scheduling problems had been on single shift and multiple shift scheduling of a single category of employees. For example, Burns and Carter (1985) considered a single shift scheduling problem that generalized the research of Brownell and Lowerre (1976). Other related single category employee scheduling problems were investigated by Lowerre (1997), Baker and Magazine (1997), Burns (1978, 1981), Baker, Burns, and Carter (1979), Emmons (1985), and Burns and Koop (1987).

A related problem to the one being addressed in this paper, was considered by Narasimhan (1996) who examined a single shift and work center scheduling of a hierarchical workforce and devised an algorithm that provides a computational scheme for arriving at a minimum-cost workforce mix of categories of employees when the demand is characterized by different levels for weekdays and weekends. This research effort extends the work of Narasimhan (1996) by including multiple shifts and work centers, and taking into consideration employees' desired preferences for shifts, work centers, and off-days. Also, this paper extends the effort of the authors in (Al-Yakoob and Sherali, 2007a), which considers a multiple shift and multiple work centers employee scheduling problem that is concerned with assigning employees to gas stations in a case study related to the Kuwait National Petroleum Corporation. Two types of employees were considered in (Al-Yakoob and Sherali, 2007a); cashiers and supervisors, who are respectively required for the self-service and full-service stations. The problem considered in this paper is similar to that studied in (Al-Yakoob and Sherali, 2007a) in the sense that both problems consider multiple shifts and multiple work centers; however, the problem of this paper involves a hierarchical employee structure having downward substitutability. Hence, different models and algorithms need to be developed. For further details on employee scheduling and related models, the reader may refer to (Alfares, 2004; Ernst $e t$ al., 2004a; Ernst et al., 2004b).

The remainder of this paper is organized as follows. The next section addresses the employees' preferences and presents some preliminary modeling notation. A mixed- 
integer programming model is formulated for the problem in Section 4. Due to the overwhelming size of this model based on a number of test problem instances, we next devise a specialized heuristic for the problem and illustrate this method by an example in Section 5. Computational results are presented in Section 6. Finally, Section 7 provides a summary along with some concluding remarks.

\section{Problem Description}

In this section, the preferences of employees, notation, and assumptions are presented in order to formulate the required mixed-integer program in Section 3.

\subsection{Preferences of Employees}

A proper representation of employees' expressed preferences for specific shifts, work centers, and weekly off-days is essential in seeking a fair work assignment for each set of employees that belong to the same category. Manual approaches to employee scheduling problems are often biased and lead to discrepancies in satisfaction levels among employees. Therefore, it is important to set some guidelines for employees' preferences, based on which, efficient and fair schedules can be generated. Below, we present such guidelines for employees' preferences that are similar to those employed by the authors in (Al-Yakoob and Sherali, 2007a).

\section{A) Preferences for off-days}

Every employee establishes a one-to-one matching between the days of the week and the list $\{1,2,3,4,5,6,7\}$. A day matched to number " 1 " indicates that this day is the employee's first off-day preference, and a day matched to number " 2 " indicates that this day is the employee's second off-day preference, and so on.

\section{B) Preferences for shifts}

Every employee is instructed to submit a permutation of the set $\{1,2,3\}$ to represent the preference with respect to daily shifts. For example, $\{3,1,2\}$ indicates that Shift 3 (10:00 p.m. $-6: 00$ a.m.) is the first choice of preference, Shift 1 (6:00 a.m. $-2: 00$ p.m.) is the second choice of preference, and Shift 2 (2:00 p.m - 10:00 p.m.) is the third choice of preference.

\section{C) Preferences for work centers}

Each employee is instructed to list, in ascending order, $m$ different work centers, the first of which represents the highest preference and the $m$ th of which represents the lowest preference, where $m$ is some positive integer to be determined based on sensitivity analyses. For later use, we associate the number " 1 " with the first choice, " 2 " with the second choice, and so on. Note that the first work center preference in the list is not necessarily the nearest one to the employee's residence; it is left to the employee to decide upon these preference choices, so long as there is a compatible need at the selected centers. 


\section{Model Formulation and Related Issues}

The requisite integer variables and constraints that will be used to formulate our class scheduling problem are presented in Sections 4.1 and 4.2, respectively. In Section 4.3, we formulate a mathematical model, and in Section 4.4, we propose a partitioning scheme to solve this model. A concise list of notation used in our analysis is provided below.

$D$ : duration of the time-horizon, which is assumed to be a multiple of weeks;

$N$ : number of weeks in the time-horizon;

$\Gamma=\{1, \ldots, D\}:$ set of days in the time-horizon;

$\Gamma_{i} \subseteq \Gamma$ : set of days that represent the $i$ th week of the time horizon, for $i=1, \ldots, N$;

$\Gamma^{W D} \subseteq \Gamma$ and $\Gamma^{W E} \subseteq \Gamma$ : respectively, subsets of weekdays and weekends in $\Gamma$;

$\Gamma_{i}^{W D} \equiv \Gamma_{i} \cap \Gamma^{W D}$ and $\Gamma_{i}^{W E} \equiv \Gamma_{i} \cap \Gamma^{W E}$, for $i=1, \ldots, N$;

$\Lambda=\{1,2,3\}$ : set of working shifts, where $t=1$ denotes the 6:00 a.m. $-2: 00$ p.m shift, $t=2$ denote the 2:00 p.m. $-10: 00$ p.m. shift, and $t=3$ denote the 10:00 p.m. 6:00 a.m shift;

$A$ : number of work centers, indexed by $l \in \Pi=\{1, \ldots, A\}$;

$\bar{K}$ : employees' categories, indexed by $k \in K \equiv\{1, \ldots \bar{K}\}$;

$C_{k}$ : per week cost to hire an employee of category $k$;

$M_{k}$ : number of available employees of category $k$;

$\Phi_{k}=\left\{1, \ldots M_{k}\right\}:$ set of employees of category $K$;

$D_{k, l, t}^{W D}$ and $D_{k, l, t}^{W E}$ : respectively the demands for employees of category $k$ at work center $l$ during shift $t$ on weekdays and on weekends, for given work center $l \in \Pi$ and a given shift $t \in \Lambda$.

REMARK 1. Categories are ranked with category 1 workers at the top as the most highly qualified, and category $\bar{K}$ workers at the bottom as the least qualified, such that an employee at a higher category can perform the work of an employee at a lower category, but not vice versa. Hence, if $k_{1}, k_{2} \in K$ with $k_{1}<k_{2}$, then $C_{k_{1}}>C_{k_{2}}$. Therefore, this describes a hierarchy of categories having downward substitutability as expounded by Emmons and Burns (1985).

\section{Model Formulation}

In this section, we formulate a mixed-integer programming model that determines a minimum-cost workforce mix of the categories of employees needed to satisfy the specified demand requirements, and to assign the selected employees to shifts and work centers while specifying their off-days based on their stated preferences as discussed in Section 2.1. In Section 4.1, we introduce the decision variables for the proposed model and in Section 4.2, we formulate the problem constraints. Sections 4.3 and 4.4 then present the model objective function and the overall model formulation, respectively.

\subsection{Decision Variables}

Define the following sets of binary decision variables. 
Let

$$
\begin{gathered}
x_{k, e, d, l, t}=\left\{\begin{array}{l}
1, \text { if employee } e \in \Phi_{k} \text { is assigned to work center } l \in \Pi \\
\text { during shift } t \in \Lambda \text { on day } d \in \Gamma \\
0, \text { otherwise },
\end{array}\right. \\
w_{k, e}=\left\{\begin{array}{l}
1, \text { if employee } e \in \Phi_{k} \text { is selected during the given time-horizon, } \\
0, \text { otherwise; }
\end{array}\right.
\end{gathered}
$$

and

$$
z_{k, e, d}=\left\{\begin{array}{l}
1, \text { if } d \text { is an on-day for employee } e \in \Phi_{k}, \\
0, \text { otherwise. }
\end{array}\right.
$$

Also, let $f_{i, d, k, l, t}$ be the number of employees of category $i(\leqslant k)$ that are used on day $d \in \Gamma$ to satisfy the demand for category $k \in K$ employees at work center $l \in \Pi$ for shift $t \in \Lambda$.

\subsection{Constraints}

The various problem constraints are formulated in turn below.

\section{A) Demand requirements for weekdays and weekends}

For a given work center $l \in \Pi$ and shift $t \in \Lambda$, the demand for employees of category $k \in K$ is the same for weekdays. However, two shifts of the same day do not necessarily have the same demand. The demand for the weekends is also the same. The following constraint guarantees that there are $D_{k, l, t}^{W D}$ employee(s) of category $k$ or higher allocated for work center $l \in \Pi$ during shift $t \in \Lambda$ on day $d \in \Gamma^{W D}$.

$$
\begin{aligned}
& \left(C_{1.1 .1}\right) \sum_{i=1}^{k} f_{i, d, k, l, t}=D_{k, l, t}^{W D}, \quad \forall k \in K, d \in \Gamma^{W D}, l \in \Pi, t \in \Lambda, \\
& \left(C_{1.1 .2}\right) \sum_{i=1}^{k} f_{i, d, k, l, t}=D_{k, l, t}^{W E}, \quad \forall k \in K, d \in \Gamma^{W E}, l \in \Pi, t \in \Lambda, \\
& \left(C_{1.2}\right) \sum_{k=i}^{\bar{K}} f_{i, d, k, l, t}=\sum_{e \in \Phi_{i}} x_{i, e, d, l, t}, \quad \forall i \in K, d \in \Gamma, l \in \Pi, t \in \Lambda .
\end{aligned}
$$

Note that for a given $x$ and for each $d \in \Gamma, l \in \Pi$, and $t \in \Lambda$, these constraints constitute a transportation subproblem.

B) Workloads and on-/off-days

An employee $e \in \Phi_{k}$ may work at some center $l \in \Pi$ during shift $t \in \Lambda$ on day $d \in \Gamma$ only if this day is an on-day for this employee as enforced by the following constraint. 
Moreover, this constraint also assures that any given employee may be assigned to at most one shift per day.

$$
\left(C_{2.1}\right) \sum_{l \in \Pi} \sum_{t \in \Lambda} x_{k, e, d, l, t}=z_{k, e, d}, \quad \forall k \in K, e \in \Phi_{k}, d \in \Gamma .
$$

An employee may work for at most five shifts at the various work centers during a given week as enforced by the following constraint. Note that this constraint also ensures that every selected employee will have at least two off-days per week.

$$
\left(C_{2.2}\right) \sum_{d=i}^{i+6} z_{k, e, d} \leqslant 5 w_{k, e}, \quad \text { for } i=1+7(j-1),
$$

where $j=1, \ldots, N$ and $\forall k \in K, e \in \Phi_{k}$.

Note that this constraint also specifies the employees that will be selected during the time-horizon and hence, prescribes the workforce structure. Moreover, since the proposed model seeks to find a minimum-cost workforce mix of categories of employees, it is very likely that most employees will not have more than two off-days per week. The maximum number of consecutive on-days that may be assigned to an employee is five as discussed in Section 1. This requirement is guaranteed by the following constraint.

$$
\left(C_{2.3}\right) \sum_{d=i}^{i+5} z_{k, e, d} \leqslant 5 w_{k, e}, \quad \text { for } i=1, \ldots,(7 N-5) \text { and } \forall k \in K, e \in \Phi_{k} .
$$

If it happens that employees prefer to have their off-days on weekends, then we can accommodate this preference, to the extent possible, by introducing a special constraint guaranteeing that employees will get some weekends off over the scheduling horizon. In this case, if $n_{k}$ denotes the fewest number of off-days that are required to coincide with weekends for an employee of category $k$, then this restriction can be formulated as follows.

$$
\left(C_{2.4}\right) \sum_{d \in \Gamma^{W E}}\left(1-z_{k, e, d}\right) \geqslant n_{k}, \quad \forall k \in K, e \in \Phi_{k} .
$$

Note that the number $n_{k}$ should be carefully selected to avoid infeasibilities. One way to determine $n_{k}$ is to perform a sensitivity analyses for any given scenario. We also include the following constraint in a disaggregated form as opposed to $\left(C_{2.2}\right)$ from the viewpoint of facilitating a tighter resulting model formulation.

$$
\left(C_{2.5}\right) z_{k, e, d} \leqslant w_{k, e}, \quad \forall k \in K, e \in \Phi_{k}, d \in \Gamma .
$$

C) Avoiding the assignment of consecutive shifts to employees

Employees are not permitted to work during two consecutive shifts on any given day or over two consecutive days, either at one work center or at different work centers. The 
first case is eliminated by Constraint $\left(C_{2.1}\right)$ while the second case is ruled out by the following constraint.

$$
\left(C_{3}\right) \sum_{l \in \Pi} x_{k, e, d, l, 3}+\sum_{l \in \Pi} x_{k, e, d+1, l, 1} \leqslant w_{k, e}, \quad \forall k \in K, e \in \Phi_{k}, d: d+1 \in \Gamma .
$$

D) Comparing relative dissatisfaction levels of employees

Recall that an employee $e \in \Phi_{k}$ specifies preference values, denoted by $C_{k, e, l}^{1}$ and $C_{k, e, t}^{2}$, that respectively indicate the desirability to work at center $l \in \Pi$ and during shift $t \in \Lambda$. Let $C_{k, e, l, t}^{(1,2)}=C_{k, e, l}^{1}+C_{k, e, t}^{2}$. In a similar fashion, the preference value of associating an employee $e \in \Phi_{k}$ with an off-day $d$ is denoted by $C_{k, e, d}^{3}$. Accordingly, we define a total preference index for each employee as follows.

$$
\begin{aligned}
& \left(C_{4.1}\right) \Delta_{k, e}=\sum_{d \in \Gamma} \sum_{l \in \Pi} \sum_{t \in \Lambda} C_{k, e, l, t}^{(1,2)} x_{k, e, d, l, t}+\sum_{d \in \Gamma} C_{k, e, d}^{3}\left(w_{k, e}-z_{k, e, d}\right), \\
& \forall k \in K, e \in \Phi_{k} .
\end{aligned}
$$

Note that if employee $e$ of category $k$ is not selected, then $w_{k, e}=0$, which implies by Constraints $\left(C_{2.1}\right)$ and $\left(C_{2.2}\right)$ that $\Delta_{k, e}=0$. As mentioned in Section 2.1, the computation of preferences of employees in this paper is similar to that discussed in (Al-Yakoob and Sherali, 2007a), and hence, for a selected employee $e \in \Phi_{k}$ of category $k \in K$, we have $13 \leqslant \Delta_{k, e} \leqslant 28+5 A$ as explained in (Al-Yakoob and Sherali, 2007a). Let $\bar{\Delta}_{k}$ be a variable representing a central preference value for employees of category $k$, and let $\Delta_{\min }=13$, and $\Delta_{\max }=28+5 A$. Then the following constraints compute the absolute difference between the satisfaction levels of employees as defined in Constraint $\left(C_{4.1}\right)$ and the central preference value given by $\bar{\Delta}_{k}$.

$$
\begin{aligned}
& \left(C_{4.2}\right) v_{k, e} \geqslant \Delta_{k, e}-\bar{\Delta}_{k}-\left(1-w_{k, e}\right)\left(\Delta_{\max }-\Delta_{\min }\right), \quad \forall k \in K, e \in \Phi_{k}, \\
& \left(C_{4.3}\right) v_{k, e} \geqslant \bar{\Delta}_{k}-\Delta_{k, e}-\left(1-w_{k, e}\right)\left(\Delta_{\max }-\Delta_{\min }\right), \quad \forall k \in K, e \in \Phi_{k} .
\end{aligned}
$$

Note that if employee $e \in \Phi_{k}$ for any $k \in K$ is selected during the specified time horizon, then $w_{k, e}=1 \Rightarrow\left(1-w_{k, e}\right)\left(\Delta_{\max }-\Delta_{\min }\right)=0$. In this case, since $v_{k, e} \geqslant\left|\Delta_{k, e}-\bar{\Delta}_{k}\right|$ and the minimization objective function will have a positive coefficient associated with $v_{k, e}$, we will automatically get $v_{k, e}=\left|\Delta_{k, e}-\bar{\Delta}_{k}\right|$. On the other hand, if employee $e \in \Phi_{k}$ for any $k \in K$ is not selected (i.e., $w_{k, e}=0$ ), it is more advantageous for the objective function to have the remaining $v_{k, e}$ values equal to zero, since the corresponding optimal value for $\bar{\Delta}_{k}$ will satisfy $\Delta_{\min } \leqslant \min _{e} \Delta_{k, e} \leqslant \bar{\Delta}_{k} \leqslant$ $\max _{e} \Delta_{k, e} \leqslant \Delta_{\max }$. Hence, $\left|\Delta_{k, e}-\bar{\Delta}_{k}\right| \leqslant\left(\Delta_{\max }-\Delta_{\min }\right)$, or that the right-hand-sides of both Constraints $\left(C_{4.2}\right)$ and $\left(C_{4.3}\right)$ are nonpositive if $w_{k, e}=0$, which along with $v_{k, e} \geqslant 0$ will ensure that $v_{k, e}=0$.

\subsection{Objective Function}

The objective function of the model aims to minimize the overall cost of the selected workforce, the sum of preference indices as expressed by the individual employees with 
respect to work centers and shift choices, and with respect to desired off-days, and the sum of absolute differences in preference achieved from some (to be determined) central preference value for selected workers. The corresponding objective terms are given below with respective weight factors $\alpha_{1}, \alpha_{2}, \alpha_{3}$, and $\alpha_{4, k}, \forall k \in K$, that reflect the relative significance to the scheduler of achieving the pertinent goals. (Note that differential values of $\alpha_{4, k}$, for $k \in K$, can be used to enforce equity to a varying extent among the different categories $k \in K$.

$$
\begin{aligned}
& \alpha_{1} \sum_{k \in K} \sum_{e \in \Phi_{k}} C_{k} w_{k, e}+\alpha_{2} \sum_{k \in K} \sum_{e \in \Phi_{k}} \sum_{d \in \Gamma} \sum_{l \in \Pi} \sum_{t \in \Lambda} C_{k, e, l, t}^{(1,2)} x_{k, e, d, l, t} \\
& \quad+\alpha_{3} \sum_{k \in K} \sum_{e \in \Phi_{k}} \sum_{d \in \Gamma} C_{k, e, d}^{3}\left(w_{k, e}-z_{k, e, d}\right)+\sum_{k \in K} \alpha_{4, k} \sum_{e \in \Phi_{k}} v_{k, e} .
\end{aligned}
$$

\subsection{A Minimum-Cost Workforce Model}

The objective function and the constraints formulated in Section 4.2 yield the following mixed-integer programming model (HESM) for the hierarchical workforce scheduling problem described in the previous section.

HESM: Minimize

$$
\begin{aligned}
& \alpha_{1} \sum_{k \in K} \sum_{e \in \Phi_{k}} C_{k} w_{k, e}+\alpha_{2} \sum_{k=1}^{\bar{K}} \sum_{e \in \Phi_{k}}^{\bar{K}} \sum_{d \in \Gamma} \sum_{l \in \Pi} \sum_{t \in \Lambda} C_{k, e, l, t}^{(1,2)} x_{k, e, d, l, t} \\
& \quad+\alpha_{3} \sum_{k \in K} \sum_{e \in \Phi_{k}} \sum_{d \in \Gamma} C_{k, e, d}^{3}\left(w_{k, e}-z_{k, e, d}\right)+\sum_{k \in K} \alpha_{4, k} \sum_{e \in \Phi_{k}} v_{k, e}
\end{aligned}
$$

\section{subject to}

$$
\begin{aligned}
& \left(C_{1.1 .1}\right) \sum_{i=1}^{k} f_{i, d, k, l, t}=D_{k, l, t}^{W D}, \quad \forall k \in K, d \in \Gamma^{W D}, l \in \Pi, t \in \Lambda, \\
& \left(C_{1.1 .2}\right) \sum_{i=1}^{k} f_{i, d, k, l, t}=D_{k, l, t}^{W E}, \quad \forall k \in K, d \in \Gamma^{W E}, l \in \Pi, t \in \Lambda, \\
& \left(C_{1.2}\right) \sum_{k=i}^{\bar{K}} f_{i, d, k, l, t}=\sum_{e \in \Phi_{i}} x_{i, e, d, l, t}, \quad \forall i \in K, d \in \Gamma, l \in \Pi, t \in \Lambda, \\
& \left(C_{2.1}\right) \sum_{l \in \Pi} \sum_{t \in \Lambda} x_{k, e, d, l, t}=z_{k, e, d}, \quad \forall k \in K, e \in \Phi_{k}, d \in \Gamma, \\
& \left(C_{2.2}\right) \sum_{d=i}^{i+6} z_{k, e, d} \leqslant 5 w_{k, e}, \quad \text { for } i=1, \ldots, 7(j-1) \text { where } j=1, \ldots, N \\
& \text { and } \forall k \in K, e \in \Phi_{k}, \quad \text { for } i=1, \ldots,(7 N-5) \text { and } \forall k \in K, e \in \Phi_{k}, \\
& \left(C_{2.3}\right) \sum_{d=i}^{i+5} z_{k, e, d} \leqslant 5 w_{k, e},
\end{aligned}
$$




$$
\begin{aligned}
& \left(C_{2.4}\right) \sum_{d \in \Gamma^{W E}}\left(1-z_{k, e, d}\right) \geqslant n_{k}, \quad \forall k \in K, e \in \Phi_{k}, \\
& \left(C_{2.5}\right) z_{k, e, d} \leqslant w_{k, e}, \quad \forall k \in K, e \in \Phi_{k}, d \in \Gamma, \\
& \left(C_{3}\right) \sum_{l \in \Pi} x_{k, e, d, l, 3}+\sum_{l \in \Pi} x_{k, e, d+1, l, 1} \leqslant w_{k, e}, \quad \forall k \in K, e \in \Phi_{k}, d: d+1 \in \Gamma, \\
& \left(C_{4.1}\right) \Delta_{k, e}=\sum_{d \in \Gamma} \sum_{l \in \Pi} \sum_{t \in \Lambda} C_{k, e, l, t}^{(1,2)} x_{k, e, d, l, t}+\sum_{d \in \Gamma} C_{k, e, d}^{3}\left(w_{k, e}-z_{k, e, d}\right), \\
& \forall k \in K, e \in \Phi_{k}, \\
& \left(C_{4.2}\right) v_{k, e} \geqslant \Delta_{k, e}-\bar{\Delta}_{k}-\left(1-w_{k, e}\right)\left(\Delta_{\max }-\Delta_{\min }\right), \quad \forall k \in K, e \in \Phi_{k}, \\
& \left(C_{4.3}\right) v_{k, e} \geqslant \bar{\Delta}_{k}-\Delta_{k, e}-\left(1-w_{k, e}\right)\left(\Delta_{\max }-\Delta_{\min }\right), \quad \forall k \in K, e \in \Phi_{k}, \\
& x_{k, e, d, l, t} \in\{0,1\}, \quad \forall k \in K, e \in \Phi_{k}, d \in \Gamma, l \in \Pi, t \in \Lambda, \\
& z_{k, e, d} \in\{0,1\}, \quad \forall k \in K, e \in \Phi_{k}, d \in \Gamma, \\
& w_{k, e} \in\{0,1\}, \quad \forall k \in K, e \in \Phi_{k}, \\
& f_{i, d, k, l, t} \geqslant 0 \quad \text { and integer } \forall k \in K, i \in\{1, \ldots, k\}, d \in \Gamma, l \in \Pi, t \in \Lambda, \\
& v_{k, e} \geqslant 0, \Delta_{k, e} \geqslant 0, \forall k \in K, e \in \Phi_{k}, \\
& \Delta_{\min } \leqslant \bar{\Delta}_{k} \leqslant \Delta_{\max } .
\end{aligned}
$$

REMARK 2. Note that exists a symmetry with respect to employees in each category that have similar preference structures in terms of working shifts, work centers, and offdays. This symmetry can encumber the branch-and-bound search process (see Sherali and Smith, 2002). We could alleviate this symmetry effect by incorporating the discriminating constraint:

$$
\Delta_{k, e} \geqslant \Delta_{k, \bar{e}}, \quad \forall(e, \bar{e}) \in E_{k}, k \in K,
$$

where $E_{k}=\left\{\left(e_{1}, e_{2}\right)\right.$ : where $e_{1}<e_{2}$ are consecutive pairs in an ordered list of employees in $\Phi_{k}$ that have an identical preference structure $\}, \forall k \in K$. This is incorporated in our runs with Model HESM.

REMARK 3. The scheduling scenario examined by Narasimhan (1996) can be readily handled using Model HESM by considering a single shift and a single work center, in which case, we have $\Lambda=\{1\}$ and $\Pi=\{1\}$. The schedules generated via the algorithm proposed in (Narasimhan, 1996) assure that weekly off-days are assigned in a consecutive manner. However, Model HESM might generate an employee schedule that allocates nonconsecutive weekly off-days for employees.

PROPOSITION 1. If the integrality of the $x$-variables is enforced, then at a resulting extreme point optimum for a fixed (feasible) $x$, (a) the $z$ - and $w$-variables will be automatically integer-valued if they are relaxed continuously in the interval $[0,1]$ and (b) the $f$-variables will be automatically integer-valued if they are relaxed continuously to be nonnegative. 
Proof. Given a feasible solution with $x$ binary-valued. Then, Part (a) follows since $z$ will be automatically binary valued by $\left(C_{2.1}\right)$ if relaxed to be continuous on $[0,1]$. The integrality of the $w$-variables follows directly from $\left(C_{2.5}\right)$ and the objective function, noting that for each $k \in K, e \in \Phi_{k}, w_{k, e}$ must equal one if $z_{k, e, d}=1$ for any $d \in \Gamma$, and that $w_{k, e}=0$ is preferred by the objective function and is feasible to the remaining constraints (including attaining $\Delta_{k, e}=v_{k, e}=0$ ) whenever $z_{k, e, d}=0, \forall d \in \Gamma$. For Part (b), the integrality of the $f$-variables follows from the fact that constraints $\left(C_{1.1 .1}\right),\left(C_{1.1 .2}\right)$, and $\left(C_{1.2}\right)$ define a transportation subproblem once $x$ is fixed at some binary values. This completes the proof.

\section{A Scheduling Algorithm}

A direct solution of Model HESM would be inconceivable if the problem involves a relatively large number of employees and work centers, because of the resulting overwhelming number of binary variables and constraints. Consequently, in this section, we devise an employee-scheduling algorithm based on Model HESM that efficiently staffs work centers with the required employees in an iterative manner by considering a single employee category demand requirement at each iteration.

Note that the demand for employees of Category 1 can be satisfied by employees of this category only, due to the hierarchical structure of categories as discussed in Sections 1 and 2. Assume that all work centers have been staffed with employees of this category. Then, the demand for Category 2 employees at the different work centers can be satisfied by employees from Category 2 and the unselected employees of Category 1 . Likewise, the demand for Category 3 employees at the different work centers can be satisfied by employees from Category 3 and the unselected employees of Categories 1 and 2. Proceeding in this fashion, the demand for Category $\bar{K}$ can be satisfied by employees of category $\bar{K}$ and the unselected employees from categories $1, \ldots,(\bar{K}-1)$. The algorithm generates an employee schedule in this manner for the first week of the time horizon $\left(\Gamma_{1}\right)$ and thus this schedule can be reused for the remaining weeks of the time horizon $\left(\Gamma_{2}, \ldots, \Gamma_{N}\right)$ to generate a complete schedule for the entire time horizon.

For $k=1, \ldots, \bar{K}$, the $k$ th iteration of the algorithm utilizes a modified version of Model HESM, denoted by HESM ${ }_{k}$ to provide work centers with the required employees of Categories $1, \ldots, k$, based on a set of employees, denoted by $\Phi_{k}^{\text {new }}$, which is generated by the algorithm. The set $\Phi_{k}^{\text {new }}$ is defined by $\Phi_{k}^{\text {new }}=\Phi_{k} \cup \Psi \cup \Omega$, where $\Phi_{k}$ is as defined in Section 2.2, $\Psi$ is a subset of employees in $\bigcup_{i=1}^{(k-1)} \Phi_{i}$ who have not been assigned any shift in iterations $1, \ldots,(k-1)$, and $\Omega$ is the set of employees who have been selected in the iterations $1, \ldots,(k-1)$, but have been assigned a total work load of less than five shifts. Let $\alpha=|\Psi|$ be the number of employees in $\Psi$ and $\beta=|\Omega|$ be the number of employees in $\Omega$. Let $a=\left|\Phi_{k}\right|+\alpha+\beta$. Let $e=1, \ldots, a$ index the employees in the set $\Phi_{k}^{\text {new }}$, where $e=1, \ldots,\left|\Phi_{k}\right|, e=\left|\Phi_{k}\right|+1, \ldots,\left|\Phi_{k}\right|+\alpha$, and $e=\left|\Phi_{k}\right|+\alpha+1, \ldots,\left|\Phi_{k}\right|+\alpha+\beta$ 
respectively index the employees in the sets $\Phi_{k}, \Psi$, and $\Omega$. Let $C_{e}^{k}$ be the cost of employee $e \in \Phi_{k}^{\text {new }}$ at iteration $k$, which is defined as follows:

$$
C_{e}^{k}=\left\{\begin{array}{l}
C_{k}, \text { if } e \in \Phi_{k} \\
0, \quad \text { if } e \in \Omega \\
C_{i}, \text { if } e \in \Psi \cap \Phi_{i} \text { where } i \in\{1, \ldots,(k-1)\}
\end{array}\right.
$$

Note that the cost of employee $e \in \Omega$ at iteration $k$ is zero, since this employee has already been selected during some previous iterations.

The decision variables of Model HESM are also used in Model $\operatorname{HESM}_{k}$, however, without the explicit reference to specific categories. These variables are modified as follows. (Note also that the flow variables $f$ in Model HESM are no longer needed in Model $\operatorname{HESM}_{k}$ because we are considering a single demand node for category $k$ in the transportation subproblems for each $d \in \Gamma, l \in \Pi$, and $t \in \Lambda$. Hence the $x$-variables themselves account for these flows directly.

$$
\begin{gathered}
x_{e, d, l, t}=\left\{\begin{array}{c}
1, \text { if employee } e \in\{1, \ldots, a\} \text { is assigned to work center } l \in \Pi \\
\text { during shift } t \in \Lambda \text { on day } d \in \Gamma, \\
0, \text { otherwise }
\end{array}\right. \\
w_{e}=\left\{\begin{array}{l}
1, \text { if employee } e \in\left\{1, \ldots,\left|\Phi_{k}\right|+\alpha\right\} \text { is selected, } \\
0, \text { otherwise }
\end{array}\right.
\end{gathered}
$$

and

$$
z_{e, d}=\left\{\begin{array}{l}
1, \text { if } d \text { is an on-day for employee } e \in\{1, \ldots, a\} \\
0, \text { otherwise. }
\end{array}\right.
$$

Note that for the first iteration of the algorithm (i.e, $k=1$ ) we have $\Omega=\Psi=\emptyset$. Now, for $e \in \Omega$, let $\bar{\Gamma}_{1, e} \subseteq \Gamma_{1}$ denote the set of fixed or known on-days associated with employee $e$ being determined based on the specific days during which shifts are assigned to employee $e$ in iterations $1, \ldots,(k-1)$, and let $\Gamma_{1, e}$ be the complement of $\bar{\Gamma}_{1, e}$ in $\Gamma_{1}$. Let $\gamma_{e}=\left|\bar{\Gamma}_{1, e}\right|$ and let $\Omega_{d} \subseteq \Omega$ be defined as follows: $e \in \Omega_{d}$ if $d \in \Gamma_{1, e}$. For $e \in \Omega$, let $\Delta_{e}^{\text {old }}$ be the satisfaction level of employee $e$ with respect to the assigned shifts, work centers, and on-days as obtained from iterations $1, \ldots,(k-1)$.

Next, we present Model $\mathrm{HESM}_{k}$ and then introduce the proposed employee scheduling algorithm (ESA). (Below, since we are considering only the days in $\Gamma_{1}$, but we are assuming a cyclic schedule, Day 8 is assumed to coincide with Day 1, and Day 0 with Day 7.)

\section{$\operatorname{HESM}_{k}:$ Minimize}

$$
\alpha_{1} \sum_{e \in\left\{1, \ldots,\left|\Phi_{k}\right|+\alpha\right\}} C_{e}^{k} w_{e}+\alpha_{2} \sum_{d \in \Gamma_{1}} \sum_{e \in\left(\Phi_{k} \cup \Psi \cup \Omega_{d}\right)} \sum_{l \in \Pi} \sum_{t \in \Lambda} C_{e, l, t}^{(1,2)} x_{e, d, l, t}
$$




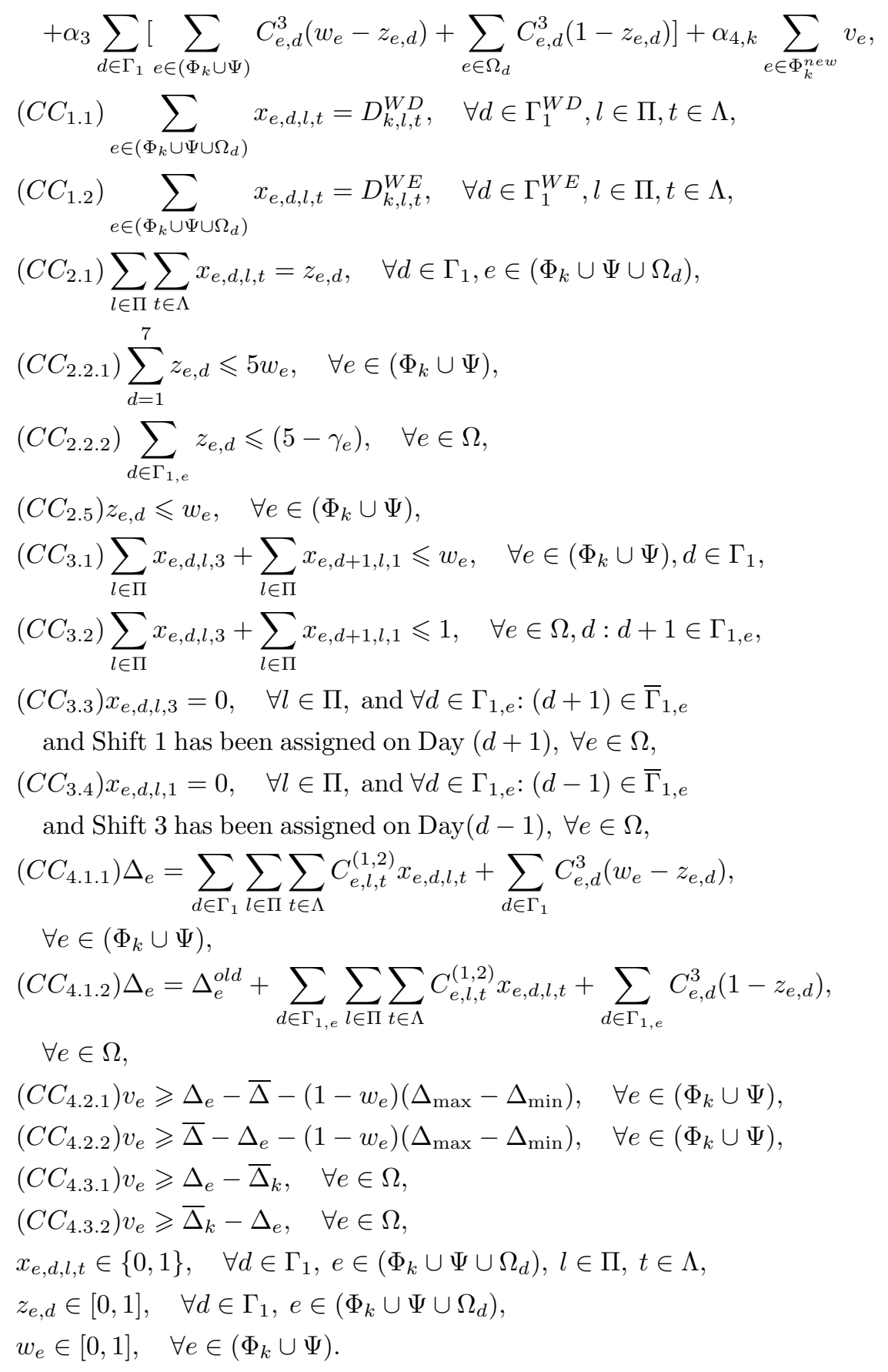

REMARK 4. The following comments relate to Model $\operatorname{HESM}_{k}$ for the $k$ th iteration of the algorithm proposed below. 
a) The right-hand-side of Constraint $\left(C C_{2.2 .1}\right)$ incorporates the binary variable $w_{e}$ because this model basically decides if an employee in the set $\left(\Phi_{k} \cup \Psi\right)$ is selected or not. On the other hand, employees in the set $\Omega$ have been already selected during some previous iteration, $1, . .,(k-1)$, and hence, the right-hand-side of Constraint $\left(C C_{2.2 .2}\right)$ ensures that an employee $e \in \Omega$ may be assigned at most $\left(5-\gamma_{e}\right)$ shifts in iteration $k$, so that the total number of shifts assigned to this employee over a week is no more than five. Also, the costs of such employees have been already factored into the total cost.

b) The satisfaction level of an employee $e \in \Omega$ after the completion of iteration $k$ is obtained by adding the satisfaction levels for shifts, work centers and off-days obtained from iterations $1, \ldots,(k-1)$ plus the satisfaction level for shifts, work centers, and offdays obtained from iteration $k$. This satisfaction level is computed by $\left(C C_{4.1 .2}\right)$. It is worth mentioning that for $e \in \Omega$, Constraints $\left(C C_{4.3 .1}\right)$ and $\left(C C_{4.3 .2}\right)$ exclude the term $\left(1-w_{e}\right)\left(\Delta_{\max }-\Delta_{\min }\right)$ because in this case, the value of $w_{e}$ is known to be 1 .

c) Model $\mathrm{HESM}_{k}$ excludes Constraints $\left(C_{2.3}\right)$ and $\left(C_{2.4}\right)$ of Model HESM because we have restricted the scheduling horizon to the first week. If the generated schedule is cyclically used for the remaining weeks of the time-horizon, then Constraint $\left(C_{2.3}\right)$ is automatically enforced. A special consideration for enforcing Constraint $\left(C_{2.4}\right)$ will be discussed later in Section 5.2.

d) Symmetry-defeating constraints similar to those given in Remark 2 can be incorporated in Model $\mathrm{HESM}_{k}$ to enhance solvability by eliminating some duplicitous solutions.

e) Note that there exists a transportation substructure in Model $\mathrm{HESM}_{k}$ that can be advantageously exploited to devise a decomposition-based solution algorithm for the employee scheduling problem under consideration. This extension is the subject of a companion follow-on paper (Sherali and Al-Yakoob, 2007).

\subsection{Algorithm ESA}

The proposed scheduling algorithm is presented next.

\section{Algorithm ESA:}

\section{Initial Step}

- Let $k=1, \Phi_{1}^{\text {new }}=\Phi_{1}, \psi=\emptyset$, and $\Omega=\emptyset$.

- Solve $\mathrm{HESM}_{1}$.

- If $\mathrm{HESM}_{1}$ is infeasible, then stop; the required staffing cannot be achieved.

- Otherwise, a solution to $\mathrm{HESM}_{1}$ yields a minimum-cost staffing for Category 1 employees.

- Fix the staffing assignments prescribed by this solution to $\mathrm{HESM}_{1}$, and denote the cost of this solution by $P$.

\section{Main Step}

- Set $k \leftarrow k+1$.

- If $k>\bar{K}$, then stop; the prescribed employee schedule is given via the successively determined solutions to Problem $\mathrm{HESM}_{k}$ for $k=1, \ldots, \bar{K}$. Otherwise, proceed as follows.

- If $\Omega \neq \emptyset$, then update $\Omega$ and its related issues as follows: 
a) Remove all employees from the set $\Omega$ that are assigned a total of five shifts.

b) For an employee $e \in \Omega$, update the set $\Gamma_{1, e} \subseteq \Gamma_{1}$ by excluding all on-days.

c) If an employee $e \in \Omega$ is assigned shift $t=3$ of some day $d \in \bar{\Gamma}_{1, e}$ and $(d+1) \in \Gamma_{1, e}$, then block shift $t=1$ of day $(d+1)$, i.e., set $x_{e,(d+1), l, 1}=$ $0, \forall l \in \Pi$. This restriction is needed to avoid assigning consecutive shifts for employee $e$ on two consecutive days. Similarly, if an employee $e$ has been assigned to Shift $t=1$ of some day $d \in \bar{\Gamma}_{1, e}$ and $(d-1) \in \Gamma_{1, e}$, then block Shift $t=3$ of day $(d-1)$, i.e., set $x_{e,(d-1), l, 3}=0, \forall l \in \Pi$. (See constraints $\left(C C_{3.3}\right)$ and $\left(C C_{3.4}\right)$.)

d) For an employee $e \in \Omega$, update the satisfaction level of this employee in terms of shifts, work centers, and off-days, i.e., update the value of $\Delta_{e}^{\text {old }}$.

- Update $\Psi$ by removing all employees that are now assigned to at least one shift on a given day $d \in \Gamma_{1}$.

- Let $\Phi_{(k-1), 1}^{\mathrm{new}} \subseteq \Phi_{(k-1)}$ and $\Phi_{(k-1), 2}^{\mathrm{new}} \subseteq \Phi_{(k-1)}$ respectively denote the selected and unselected sets of employees of category $(k-1)$ as obtained from the solution of Model HESM $_{k-1}$.

- Let $\bar{\Phi}_{(k-1), 1}^{\text {new }} \subseteq \Phi_{(k-1), 1}^{\text {new }}$ denote the set of selected employees of category $(k-1)$ where each employee is assigned less than 5 shifts.

- Update $\Omega \leftarrow \Omega \cup \bar{\Phi}_{(k-1), 1}^{\text {new }}$ and $\Psi \leftarrow \Psi \cup \Phi_{(k-1), 2}^{\text {new }}$.

- Set $\Phi_{k}^{\text {new }}=\Phi_{k} \cup \Psi \cup \Omega$.

- Solve $\mathrm{HESM}_{k}$ : If $\mathrm{HESM}_{k}$ is infeasible, then stop; the heuristic has been unable to achieve the required staffing. Else, fix the staffing assignments made thus far, and update the total cost $P$ and repeat the Main Step.

REMARK 5. Algorithm ESA generates a minimum-cost employee schedule for the first week of the time-horizon, which incorporates employees' preferences for shifts, work centers, and off-days. To generate an employee schedule for the entire time-horizon, we repeat the same schedule obtained via Algorithm ESA for the remaining weeks $\Gamma_{2}, \ldots, \Gamma_{N}$ in the time-horizon. Such a schedule automatically satisfies Constraint $\left(C_{2.3}\right)$. However, the resulting schedule might be unsatisfactory for employees who have not been assigned any off-days coinciding with weekends. Note that since Constraint $\left(C_{2.4}\right)$ might not be satisfied for the entire time-horizon, we can better accommodate this constraint via a rolling horizon algorithm, which generates weekly schedules using Algorithm ESA and attempts to satisfy Constraint $\left(C_{2.4}\right)$ to the extent possible.

A simple example to elucidate the approach of Algorithm ESA can be gleaned from the website: www.al-yakoob.com.

\section{Computational Results}

In this section, we present computational results for Model HESM and Algorithm ESA based on a set of ten test problems. Table 1 presents statistics related to these problems, denoted by $P_{1}, \ldots, P_{10}$. These test problems represent different scheduling scenarios for a time-horizon of $N=1$ week and having three shifts per day. The demand for employees 
Table 1

Test problems

\begin{tabular}{cccc}
\hline$P_{i}$ & $\begin{array}{c}\text { Number of } \\
\text { employee categories }\end{array}$ & $\begin{array}{c}\text { Number of } \\
\text { work centers } \\
|A|\end{array}$ & $\left\{\left|\Phi_{1}\right|,\left|\Phi_{2}\right|, \ldots,\left|\Phi_{\bar{K}}\right|\right\}$ \\
\hline 1 & $\bar{K}$ & 1 & $\{5,7,5,6\}$ \\
2 & 4 & 1 & $\{5,7,5,6,5\}$ \\
3 & 5 & 2 & $\{14,13\}$ \\
4 & 2 & 2 & $\{14,13,13\}$ \\
5 & 3 & 3 & $\{14,13,13,12\}$ \\
6 & 4 & 3 & $\{17,14\}$ \\
7 & 2 & 4 & $\{17,14,15\}$ \\
8 & 3 & 4 & $\{17,14,15,15,15\}$ \\
9 & 4 & 4 & $\{22,19\}$ \\
10 & 5 & 4 & \\
\hline
\end{tabular}

of a given category during any shift at a work center is either one, two, or three. The employees' preferences for work centers, shifts, and off-days were generated randomly. The parameters $\alpha_{1}, \alpha_{2}, \alpha_{3}$, and $\alpha_{4, k}, \forall k \in K$, were all set to a value of 1 . (Note that these parameters reflect the relative importance of achieving the pertinent objectives as discussed in Section 4.3.) Furthermore, for the (up to) five employee categories considered, respective values of $C_{k}$ for $k=1, \ldots, 5$ were taken as 800, 700,600, 500, and 450 . All runs were made on a Pentium 4, CPU $1.70 \mathrm{GHz}$ computer having $1024 \mathrm{MB}$ of RAM using CPLEX-9.0, with coding in Java.

Let $\overline{\mathrm{HESM}}$ denote the linear relaxation of Model HESM and let $v(\mathrm{M})$ denote the optimal objective function value of any model M. Also, let $V(\mathrm{ESA})$ denote the objective function value obtained via using Algorithm ESM. Table 2 presents results related to

Table 2

Results related to solving model $\overline{\mathrm{HESM}}$

\begin{tabular}{cccccc}
\hline \multirow{2}{*}{\begin{tabular}{c}
$P_{i}$ \\
\cline { 1 - 2 } Row
\end{tabular}} & Column & Non-zero entry & $\begin{array}{c}\text { CPU time } \\
\text { (seconds) }\end{array}$ & $v \overline{(\mathrm{HESM})}$ \\
\hline 1 & 568 & 703 & 4107 & 0.01 & 14528.40 \\
2 & 710 & 929 & 5142 & 0.01 & 16860.40 \\
3 & 819 & 1448 & 6036 & 0.01 & 18134.24 \\
4 & 1212 & 2209 & 9064 & 0.01 & 26403.80 \\
5 & 1620 & 3002 & 12040 & 0.02 & 31450.62 \\
6 & 972 & 2328 & 9289 & 0.02 & 23137.98 \\
7 & 1440 & 3550 & 13966 & 0.02 & 32420.84 \\
8 & 1971 & 4898 & 18895 & 0.03 & 40427.10 \\
9 & 2502 & 6309 & 23950 & 0.04 & 47654.76 \\
10 & 1287 & 3940 & 15362 & 0.02 & 31264.07 \\
\hline
\end{tabular}


solving Model $\overline{\mathrm{HESM}}$ using the CPLEX Optimization Package (version 9.0).

Let perct_opt $(v(H E S M), V(E S M))=100\left(1-\frac{V(\mathrm{ESA})-v(\overline{\mathrm{HESM}})}{V(\mathrm{ESA})}\right)$. Table 3 presents statistics related to solving the employee scheduling problem using Algorithm ESA.

\section{REMARK 6.}

a) We attempted to solve Model HESM using CPLEX with optimality gap set at ten percent. For all test problems, we were unable to solve Model HESM due to out-of-memory problems.

b) Using the default CPLEX optimality gap in all the iterations of Algorithm ESA, we were able to obtain solutions for all the test problems within at least 92.30 percent of optimality (see Table 6).

c) The largest number of work centers considered in the test problems was four, namely for $P_{i}, i=7, \ldots, 10$. For test problems having significantly more work centers, the number of variables and constraints in Models HESM is expected to be high, in which case, solutions for such scheduling scenarios might be out-ofreach, even via Algorithm ESA. In such cases, however, we can utilize a two-stage approach similar to the one proposed by the authors in (Alfares, 2004; Al-Yakoob and Sherali, 2007a) in concert with Algorithm ESA. In the first stage, mutually exclusive subsets of employees can be matched to mutually exclusive subsets of work centers based on employees' expressed preferences for work centers (and secondarily, for shift 3 , in particular, which is typically an undesirable shift and, hence, a discerning factor). Then, in the second stage, employees can be efficiently assigned to shifts in order to satisfy demand requirements at the work centers (and to decide upon their off-days, by applying Algorithm ESA to each subproblem created by the matched sets of employees and work centers.

Table 3

Statistics related to solving the employee scheduling problem using Algorithm ESA

\begin{tabular}{cccc}
\hline $\begin{array}{c}\text { Test problem } \\
P_{i}\end{array}$ & V(ESA) & $\begin{array}{c}\text { Total } \\
\text { CPU Time } \\
\text { (seconds) }\end{array}$ & perct_opt $(v(H E S M), V(E S M))$ \\
\cline { 1 - 3 } 1 & & 0.05 & 92.82 \\
2 & 15651 & 0.06 & 92.30 \\
3 & 18139 & 22.03 & 97.00 \\
4 & 18695 & 123.45 & 98.19 \\
5 & 26891 & 123.45 & 97.90 \\
6 & 32123 & 0.18 & 99.97 \\
7 & 23144 & 0.26 & 99.97 \\
8 & 32429 & 0.33 & 98.52 \\
9 & 41033 & 24.07 & 98.80 \\
10 & 48232 & 159.70 & 98.59 \\
\hline
\end{tabular}




\section{Summary and Concluding Remarks}

This paper is concerned with a hierarchical workforce employee scheduling problem involving multiple shifts and work centers. In a hierarchical workforce structure, an employee in a higher category can perform the duties of an employee belonging to a lower category, but not vice versa. However, a higher category worker is relatively more costly. A mixed-integer programming model for the problem that generates a minimum-cost employee schedule while taking into consideration employees' stated preferences for shifts, work centers, and off-days has been developed. A heuristic scheduling algorithm that sequentially utilizes a simplified version of this model and staffs work centers with available employees of a given category or higher at each iteration is proposed. This algorithm can be utilized to solve problems that cannot be solved directly via the initial formulation. In fact, we were unable to solve Model HESM for any of our ten test problems directly via CPLEX. Algorithm ESA, however, enabled us to solve these instances having 76 employees of up to five categories and four work centers to within at least 92.30 percent of optimality, within 2.66 CPU minutes on a Pentium 4, CPU $1.70 \mathrm{GHz}$ computer having $1024 \mathrm{MB}$ of RAM. The results indicate that cost effective and fair employee schedules that incorporate preferences for shifts, work centers, and off-days can be efficiently generated in a timely fashion using this procedure. Furthermore, this modeling approach can be readily combined with the two-stage approach developed by the authors in (Al-Yakoob and Sherali, 2007a; Al-Yakoob and Sherali, 2007b)) to handle problems having a relatively greater number of work centers and employees.

\section{References}

Alfares, H. (2004). Survey, categorization, and comparison of recent tour scheduling literature. Annals of Operations Research, 127, 145-175.

Al-Yakoob, S.M., and H.D. Sherali (2007a). Mixed-integer programming models for an employee scheduling problem with multiple shifts and work locations. Annals of Operation Research (accepted).

Al-Yakoob, S.M., and H.D. Sherali (2007b). Minimum Weighted Matching-Based Partitioning of a Complete Bipartite Graph. Department of Mathematics and Computer Science, College of Science, Kuwait University.

Baker, K., and M. Magazine (1977). Workforce scheduling with cyclic demands and day off constraints. Management Science, 24, 161-167.

Baker, K., R. Burns and M. Carter (1979). Staff scheduling with day-off and workstretch constraints. AIIE Transactions, 6(11), 286-292.

Brownell, W., and J. Lowerre (1976). Scheduling of work forces required in continuous operations under alternative labor policies. Management Science, 22, 297-305.

Burns, R. (1978). Manpower scheduling with variable demands and alternate weekends off. Information, 16, $101-111$.

Burns, R. (1981). An iterative approach to multiple shift scheduling. Manuscript, School of Business, Queen's University, Kingston, Ontario, Canada.

Burns, R., and M. Carter (1985). Work force size and schedules with variable demands. Management Science, 31, 599-607.

Burns, R., and G. Koop (1987). A modular approach to optimal multiple-shift manpower scheduling. Operations Research, 35, 100-110.

Emmons, H. (1985). Workforce scheduling with cyclic requirements and constraints on days, off weekends, and workstretch. IIE Transactions, 17, 8-16. 
Emmons, H., and R. Burns (1991). Off-day scheduling with hierarchical worker categories. Operations Research, 39, 484-495.

Ernst, A.T., H. Jiang, M. Krishnamoorthy, B. Owens and D. Sier (2004a). An annotated bibliography of personnel scheduling and rostering. Annals of Operations Research, 127, 21-144.

Ernst, A.T., H. Jiang, M. Krishnamoorthy and D. Sier (2004b). A review of applications, methods, and models. European Journal of Operational Research, 153, 3-27.

Lowerre, J. (1977). Workstretch properties for the scheduling of continuous operations under alternative labor polices. Management Science, 23, 963-971.

Narasimhan, R. (1996). An algorithm for single shift scheduling of hierarchical workforce. European Journal of Operational Research, 96, 113-121.

Sherali, H.D., and J.C. Smith (2002). Improving discrete model reorientations via symmetry considerations. Management Science, 47(10), 1396-1407.

Sherali, H.D., and S.M. Al-Yakoob (2007). A Transportation Based Approach for an Employee Scheduling Problem. Department of Mathematics and Computer Science, College of Science, Kuwait University.

S. Al-Yakoob is an associate professor at the Department of Mathematics and Computer Science at Kuwait University. His research interests include mathematical programming and optimization with applications to real world problems such as location, transportation, scheduling, and financial problems.

H.D. Sherali is a university distinguished professor and the W. Thomas Rice endowed chaired professor of engineering in the Industrial and Systems Engineering Department at Virginia Polytechnic Institute and State University. His areas of research interest are in analyzing problems and designing algorithms for specially structured linear, nonlinear, and integer programs arising in various applications, global optimization methods for non-convex programming problems, location and transportation theory and applications, economic and energy mathematical modeling and analysis. He has published over 238 refereed articles in various operations research journals, has (co-) authored six books in this area, and serves on the editorial board of eight journals. He is an elected member of the National Academy of Engineering.

\title{
Tvarkaraščiu sudarymas daugelio pamainụ ir darbo centru uždaviniuose, kai darbo jèga hirarchinè
}

\author{
Salem M. AL-YAKOOB, Hanif D. SHERALI
}

Straipsnyje nagrinejjamas tvarkaraščių sudarymas daugelio pamainų ir darbo centrų uždaviniuose, kai naudojama daugelio kategorijų darbo jẻga. Aukštesnès kategorijos darbuotojai gali atlikti žemesnès kategorijos darbą, bet ne atvirkščiai. Todèl aukštesnès kategorijos darbuotojas gauna didesnị atlyginimą. Apkrovimas tas pats visoms darbo dienoms, tačiau gali skirtis savaitgaliais. Siekiama minimizuoti išlaidas atsižvelgiant ị darbuotoju pageidavimus. Šiam uždaviniui spręsti siūlomas euristinis algoritmas. Sulyginimas su tiksliu dalinai sveikaskaitinio tiesinio programavimo algoritmu rodo, kad optimalus sprendinys randamas $92-98 \%$ atvejų. 